\title{
EFICIÊNCIA DA ESTABILIZAÇÃO DO SOLO E QUALIDADE DE TIJOLOS PRENSADOS DE TERRA CRUA TRATADA COM ADITIVOS QUÍMICOS, AVALIADAS PELA COMBINAÇÃO DE TESTES DESTRUTIVOS E NÃO-DESTRUTIVOS
}

\author{
RÉGIS DE C. FERREIRA ${ }^{1}$, WESLEY J. FREIRE ${ }^{2}$
}

\begin{abstract}
RESUMO: A qualidade de tijolos prensados de terra crua tratada quimicamente é influenciada basicamente pelo tipo de solo, adições químicas e período de cura. O presente trabalho teve como objetivo estudar a combinação de métodos destrutivos e não-destrutivos associados à análise estatística, para a avaliação da qualidade e da eficiência da estabilização de tijolos prensados de terra crua tratada com cimento, cal e silicato de sódio. Os teores de cimento e cal foram 0; 6 e 10\%, e a dose de silicato de sódio foi de $4 \%$ em relação ao peso seco da mistura solo-aditivo. Após a sua moldagem, os tijolos foram submetidos à cura durante os períodos de 7; 28; 56 e 91 dias. As propriedades físicomecânicas dos tijolos foram determinadas por meio de testes destrutivos, tais como a resistência à compressão simples e a absorção de água, e não-destrutivos por meio do ensaio acústico do ultra-som. Adotou-se o parâmetro "resistência anisotrópica" para simplificar as interpretações estatísticas. A adição química que conferiu a melhor qualidade técnica aos tijolos, foi a de $10 \%$ de cimento. O parâmetro resistência anisotrópica mostrou-se promissor com vistas à avaliação da qualidade técnica dos tijolos.
\end{abstract}

PALAVRAS-CHAVE: tijolos de terra crua, propriedades mecânicas, material de construção.

\section{EFFICIENCY OF SOIL STABILIZATION AND QUALITY OF BRICKS MANUFACTURED WITH SOIL ADDED WITH CHEMICAL ADDITIVES AND EVALUATED THROUGH THE ASSOCIATION OF DESTRUTIVE AND NON-DESTRUCTIVE METHODS}

SUMMARY: The aim of this research was the studying of the efficiency of soil stabilization and the technical quality of bricks manufactured with two types of soil treated with chemical additives. For this purpose a sandy soil and a clayey one were added of Portland cement, lime and sodium silicate being their mechanical characteristics evaluated through both non-destructive and destructive methods. The Portland cement and lime admixture contents were 0; 6 and 10\%, and the sodium silicate dosage was $4 \%$. Those two methods were associated in order to describe precisely a quantitative parameter called "anisotropical resistance". The results showed that such a parameter could be used as a good index for brick's technical quality evaluation.

KEYWORDS: stabilized soil bricks, mechanical properties, construction material.

\section{INTRODUÇÃO}

Já há algum tempo, devido às crises econômica, habitacional e, mais recentemente, energética pelas quais passa o Brasil, pesquisadores vêm resgatando técnicas e materiais que possam oferecer soluções apropriadas para a construção de moradias de interesse social, urbanas ou rurais.

Face a isso, a partir da segunda metade da última década, tem havido, em âmbito mundial, considerável aumento no número e na qualidade das pesquisas voltadas ao estudo do solo como

\footnotetext{
${ }^{1}$ Eng ${ }^{\circ}$ Agrônomo, Prof. Adjunto, Escola de Agronomia e Engenharia de Alimentos, UFG, Câmpus Samambaia, s/nº , Goiânia - GO, Fone: (0XX62) 521.1534, e-mail: rcastro@agro.ufg.br

${ }^{2}$ Prof. Titular, Departamento de Construções Rurais, FEAGRI/UNICAMP, Campinas - SP.

Recebido pelo Conselho Editorial em: 2-6-2003

Aprovado pelo Conselho Editorial em: 11-8-2004
} 
material de construção. Assim, grandes progressos têm sido alcançados com a finalidade de melhorar as características do solo por meio de sua estabilização com aditivos químicos, tais como o cimento, a cal, a álcalis fortes, as cinzas volantes, as escórias de alto forno, dentre outros (GEYER et al., 1995; FREIDIN \& ERELL, 1995; WALKER, 1995; BARBOSA \& MATTONE; GORDON et al., 1996; NGOWI, 1997; BARÔNIO \& BINDA, 1997; KANIRAJ \& HAVANAGI, 1999; ROLIM et al., 1999; FERREIRA \& CAMARINI, 2001; FERREIRA \& FREIRE, 2003).

A cal e o cimento são aditivos químicos bastante empregados com vistas à estabilização química de solos. Por sua vez, o tratamento do solo com álcalis fortes favorece as reações do quartzo com os estabilizadores alcalinos, tais como a cal e o cimento Portland, podendo a resistência inicial ser elevada em 15 a $400 \%$. O silicato de sódio $\left(\mathrm{Na}_{2} \mathrm{SiO}_{3}\right)$, o carbonato de sódio $\left(\mathrm{Na}_{2} \mathrm{CO}_{3}\right)$ e o hidróxido de sódio $(\mathrm{NaOH})$ são álcalis usualmente associadas ao cimento e à cal com a finalidade de estabilização de solos (FERREIRA \& FREIRE, 2003). O silicato de sódio é usado na estabilização de solos, principalmente porque ele reage com os sais solúveis de cálcio em soluções aquosas para formar silicatos de cálcio gelatinosos insolúveis. Os silicatos de cálcio hidratados são agentes cimentantes e melhoram a estabilidade do solo, preenchendo seus vazios e expulsando, conseqüentemente, a água do solo (REN \& KAGI, 1995).

Dessa forma, a presença ou não de aditivos, a granulometria e a classificação do solo, e as condições de cura são importantes fatores que influenciam na estabilização e, conseqüentemente, na qualidade dos tijolos prensados de terra crua.

Por sua vez, a avaliação do grau de estabilização de tijolos prensados de terra crua, adicionada ou não de aditivos, é feita, comumente, por meio de testes físico-mecânicos destrutivos, tais como ensaios de compressão simples e capacidade de absorção de água. No Brasil, a NBR 08491 (ABNT, 1992b) é a responsável pela normatização dos valores aceitáveis para esses parâmetros para tijolos maciços de solo-cimento, recomendando resistência mínima de $2 \mathrm{MPa}$ e valor máximo de $20 \%$ de absorção de água, ambos avaliados aos sete dias após a moldagem dos tijolos.

Além dos testes destrutivos, algumas caracterizações podem ser realizadas por intermédio de testes não-destrutivos. No caso do concreto e da madeira, o método acústico do ultra-som já vem sendo utilizado há vários anos para a determinação de diversos parâmetros, tais como resistência à compressão simples e módulo de elasticidade dinâmico (BERALDO, 2002).

O método do ultra-som é baseado na introdução de ondas ultra-sônicas por meio de pulsos periódicos em frequiências entre 20 e $100 \mathrm{kHz}$, usando um transdutor (emissor) acoplado à superfície. Após a propagação da onda através do material, a mesma é coletada por outro transdutor (receptor). O tempo $(\mu \mathrm{s})$ decorrido da emissão à recepção é então medido, obtendo-se a velocidade $\left(\mathrm{m} \mathrm{s}^{-1}\right)$. Para $\mathrm{o}$ caso de tijolos, CULTRONE et al. (2001) afirmaram que a velocidade da onda ou pulso ultra-sônico é influenciada pela sua composição mineralógica, estrutura cristalina, massa específica aparente e teor de água. Já a presença de vazios, fissuras e rachaduras promovem queda na velocidade como resultado da absorção e dispersão do pulso ultra-sônico. Determinada a velocidade, tem-se uma idéia sobre a qualidade, uniformidade e resistência do material (QASRAWI, 2000).

CULTRONE et al. (2001) propuseram que os estudos da qualidade técnica de materiais de construção poderiam ser preferencialmente executados mediante o uso de testes não-destrutivos. Sendo assim, técnicas baseadas na propagação da onda ultra-sônica são obviamente vantajosas.

Dentro desse contexto, o objetivo do presente trabalho foi o de estudar a viabilidade da combinação de métodos destrutivos e não-destrutivos associados à análise estatística, para a avaliação da qualidade e da eficiência da estabilização de tijolos prensados de terra crua tratada com cimento, cal e silicato de sódio. A fim de simplificar a interpretação estatística dos dados, foi adotado o parâmetro "resistência anisotrópica" definido por CULTRONE et al. (2001), ao avaliarem a qualidade de tijolos 
queimados produzidos a partir de diferentes matérias-primas e temperaturas, e calculado a partir dos resultados obtidos dos testes mecânicos e ultra-sônicos.

\section{MATERIAL E MÉTODOS}

Foram estudados dois tipos de solo, de duas classes texturais distintas, ou seja, um solo de textura granular $\left(\mathrm{A}_{2-4(0)}\right)$ e outro de textura fina $\left(\mathrm{A}_{5(6)}\right)$ (Tabela 1). Foram usados cimento Portland (CPII E 32) e cal hidratada (CH-I). As características físico-químicas do cimento e da cal seguiram a NBR 11578 (ABNT, 1991) e a NBR 7175 (ABNT, 1992a), respectivamente. Foi usado silicato de sódio alcalino comercial com $37 \%$ de $\mathrm{SiO}_{2}$ e $16 \%$ de $\mathrm{Na}_{2} \mathrm{O}$ e relação sílica:álcali de 2,3:1.

TABELA 1. Características físico-mecânicas dos solos utilizados.

\begin{tabular}{|c|c|c|}
\hline Índices Físicos* & Solo Granular & Solo Fino \\
\hline Umidade natural $(\%)$ & 3,75 & 17,01 \\
\hline Massa específica dos sólidos $\left(\mathrm{g} \mathrm{cm}^{-3}\right)$ & 2,65 & 2,90 \\
\hline Limite de Liquidez (\%) & $\mathrm{NP} * *$ & 44,67 \\
\hline Limite de Plasticidade (\%) & NP & 36,28 \\
\hline Índice de Plasticidade (\%) & NP & 8,39 \\
\hline \multicolumn{3}{|c|}{ Distribuição Granulométrica (\%) } \\
\hline Pedregulho (>2 mm) & 0,00 & 0,00 \\
\hline Areia $(0,05-2 \mathrm{~mm})$ & 68,35 & 39,35 \\
\hline Silte $(0,005-0,05 \mathrm{~mm})$ & 20,25 & 30,55 \\
\hline $\operatorname{Argila}(<0,005 \mathrm{~mm})$ & 11,50 & 30,10 \\
\hline \multirow[t]{2}{*}{ Porcentagem que passa na peneira 200} & 33,30 & 63,80 \\
\hline & \multicolumn{2}{|c|}{ Compactação Proctor Normal } \\
\hline \multirow{4}{*}{$\begin{array}{c}\text { Massa específica } \\
\text { aparente seca máxima } \\
\qquad\left(\mathrm{g} \mathrm{cm}^{-3}\right)\end{array}$} & 1,96 & 1,60 \\
\hline & 1,91 & 1,57 \\
\hline & 1,83 & 1,55 \\
\hline & 1,97 & 1,62 \\
\hline $10 \%$ de cimento & 1,97 & 1,61 \\
\hline \multirow{4}{*}{$\begin{array}{l}\text { Umidade ótima de } \\
\text { compactação } \\
(\%)\end{array}$} & 11,40 & 25,05 \\
\hline & 11,67 & 25,00 \\
\hline & 13,15 & 26,60 \\
\hline & 11,14 & 24,49 \\
\hline $10 \%$ de cimento & 11,13 & 22,95 \\
\hline Descrição visual do solo & Areia siltosa & Silte areno-argiloso \\
\hline Classificação Bureau of Public Roads & Arenosa & Siltoso \\
\hline Classificação AASHTO & $\mathrm{A}_{2-4(0)}$ & $\mathrm{A}_{5(6)}$ \\
\hline Classificação USAD & Franco-arenosa & Franco-argiloso \\
\hline \multicolumn{3}{|l|}{ * Resultados de 3 repetições; ** Não-plástico. } \\
\hline \multicolumn{3}{|c|}{$\begin{array}{l}\text { Os ensaios de compressão simples foram executados em uma prensa universal (DYNATEST } \\
\text { controle de carregamento e capacidade máxima de } 10.000 \mathrm{kN} \text {. Os ensaios acústicos foran } \\
\text { izados em um aparelho portátil de ultra-som (Steinkamp/BP7, Alemanha), usando um transduto } \\
\text { ontato com freqüência de emissão do pulso ultra-sônico de } 45 \mathrm{kHz} \text {. Os tijolos foram moldados en } \\
\text { máquina de fabricação de tijolos marca SOLOTEST, de acionamento manual para a compactaçã } \\
\text { nistura fresca. Essa máquina tem capacidade de fabricação de três tijolos por prensagem, tijolo } \\
\text { II, de acordo com a NBR } 08491 \text { (ABNT, 1992b). }\end{array}$} \\
\hline
\end{tabular}


Os solos foram submetidos a ensaios de caracterização para determinar seus principais índices físicos, a saber: umidade natural (\%); distribuição granulométrica (\%), NBR 07181 (ABNT, 1984c); massa específica dos sólidos $\left(\mathrm{g} \mathrm{cm}^{-3}\right)$, NBR 07251 (ABNT, 1982); limite de liquidez (\%), NBR 06459 (ABNT, 1984a); limite de plasticidade, NBR 07180 (ABNT, 1984b); massa específica aparente seca máxima $\left(\mathrm{g} \mathrm{cm}^{-3}\right)$, e umidade ótima de compactação (\%) (ABNT, 1986).

Para a realização desta pesquisa, foram definidos os seguintes tratamentos:

T1 - solo arenoso, sem adições (testemunha);

$\mathrm{T} 2$ - solo arenoso $+6 \%$ de cimento;

$\mathrm{T} 3$ - solo arenoso $+10 \%$ de cimento;

T4 - solo arenoso $+6 \%$ de cimento $+4 \%$ de silicato de sódio;

T5 - solo arenoso $+10 \%$ de cimento $+4 \%$ de silicato de sódio;

$\mathrm{T} 6$ - solo arenoso $+6 \%$ de cal;

$\mathrm{T} 7$ - solo arenoso $+10 \%$ de cal;

T8 - solo arenoso $+6 \%$ de cal $+4 \%$ de silicato de sódio;

T9 - solo arenoso $+10 \%$ de cal $+4 \%$ de silicato de sódio;

T10 - solo siltoso, sem adições (testemunha);

$\mathrm{T} 11$ - solo siltoso $+6 \%$ de cimento;

T12 - solo siltoso $+10 \%$ de cimento;

T13 - solo siltoso $+6 \%$ de cimento $+4 \%$ de silicato de sódio;

T14 - solo siltoso $+10 \%$ de cimento $+4 \%$ de silicato de sódio;

$\mathrm{T} 15$ - solo siltoso $+6 \%$ de cal;

T16 - solo siltoso $+10 \%$ de cal;

$\mathrm{T} 17$ - solo siltoso $+6 \%$ de cal $+4 \%$ de silicato de sódio, e

T18 - solo siltoso $+10 \%$ de cal $+4 \%$ de silicato de sódio.

Os teores de $6 \%$ de cal e $6 \%$ de cimento foram escolhidos em função das recomendações de SHERWOOD (1993), que define um teor mínimo de 4\% de estabilizante para a estabilização química de solos. De acordo com o autor, para teores menores que 4\%, o termo "estabilização de solos" se torna inadequado, devendo ser adotado o termo "solo melhorado". Os teores de $10 \%$ de cal e $10 \%$ de cimento foram escolhidos em função das recomendações de ROLIM et al. (1999), que adotaram o teor de $8 \%$ de cimento para solos com características similares ao arenoso estudado. Como margem de segurança, arbitrou-se o teor de 10\%. A adição de $4 \%$ de silicato de sódio associado aos teores de cal e cimento baseou-se nas recomendações de RUFF \& DAVIDSON (1961) e FREIRE (1981).

Os teores de cimento e cal (6\% e 10\%) foram adicionados em relação ao peso do solo seco e, em seguida, a mistura solo-aditivo (cal ou cimento) foi devidamente homogeneizada. O silicato de sódio (4\%) foi tomado em relação ao peso seco da mistura solo-aditivo (cal ou cimento) e diluído na água de amassamento. A quantidade da água de amassamento correspondeu à diferença entre os teores das umidades ótima e natural do solo.

Os tijolos referentes a cada tratamento foram moldados de acordo com a norma NBR 10832 (ABNT, 1992d). Apesar de a máquina de fabricação dos tijolos possuir três fôrmas para a moldagem 
de três tijolos por vez, foi usada somente a fôrma central a fim de garantir as mesmas condições de moldagem para todos os tijolos, moldando-se, portanto, um tijolo por vez. Após a moldagem, os tijolos foram submetidos à cura em câmara úmida, em condições controladas de temperatura $\left(24{ }^{\circ} \mathrm{C} \pm 2\right)$ e umidade relativa $(90 \% \pm 2)$ por $7 ; 28 ; 56$ e 91 dias.

\section{Teste do ultra-som}

Após cada período de cura, os tijolos foram imersos em água durante $4 \mathrm{~h}$ e submetidos aos testes de ultra-som antes de serem submetidos ao ensaio de compressão simples. $\mathrm{O}$ tempo de propagação da onda ultra-sônica foi determinado por intermédio de aparelho portátil de ultra-som (Figura 1). A velocidade da onda foi calculada pela eq.(1) (QASRAWI, 2000).

$$
\mathrm{V}=\frac{\mathrm{d}}{\mathrm{t}} 1000
$$

em que,

$\mathrm{V}$ - velocidade do pulso ultra-sônico, $\mathrm{m} \mathrm{s}^{-1}$;

$\mathrm{d}$ - distância percorrida pelo pulso, $\mathrm{mm}$, e

$\mathrm{t}$ - tempo transcorrido no percurso, $\mu \mathrm{s}$.

\section{Propriedades físico-mecânicas}

Após os testes de ultra-som, os tijolos foram submetidos aos ensaios de compressão simples, de acordo com a NBR 08492 (ABNT, 1992c). Os valores foram calculados individualmente para cada tijolo. Os valores obtidos são média de três repetições, sendo a resistência à compressão simples obtida por meio da eq.(2).

$$
\mathrm{R}_{\mathrm{C}}=\frac{\mathrm{C}}{\mathrm{S}} 0,1
$$

em que,

$\mathrm{R}_{\mathrm{C}}$ - resistência à compressão simples, $\mathrm{MPa}$;

C - máxima carga, kgf, e

$\mathrm{S}$ - área, $\mathrm{cm}^{2}$.

O ensaio de absorção de água foi realizado somente aos sete dias de idade, seguindo os procedimentos da NBR 08492. Os tijolos foram levados à estufa, entre $105^{\circ} \mathrm{C}$ e $110^{\circ} \mathrm{C}$, até constância de massa, obtendo-se assim a massa do tijolo seco em estufa $\left(\mathbf{M}_{1}\right)$, em gramas. Em seguida, os tijolos foram imersos em água durante $24 \mathrm{~h}$. Após esse período, os tijolos foram retirados, enxugados superficialmente e novamente pesados, anotando-se sua massa saturada $\left(\mathrm{M}_{2}\right)$, em gramas. Os valores individuais de absorção de água (A), em porcentagem, foram obtidos pela eq.(3).

$$
\mathrm{A}=\frac{\mathrm{M}_{2}-\mathrm{M}_{1}}{\mathrm{M}_{1}} 100
$$

\section{Anisotropia total}

A anisotropia total é um parâmetro que quantifica a anisotropia da estrutura de um material, sendo calculada pela eq.(4) (CAZALLA et al., 1999).

$$
\Delta \mathrm{M}=100\left[1-\frac{2 \mathrm{~V}_{1}}{\left(\mathrm{~V}_{2}+\mathrm{V}_{3}\right)}\right]
$$

em que, 
$\Delta \mathrm{M}$ - anisotropia total, \%;

$\mathrm{V}_{1}$ - velocidade da onda ultra-sônica através da menor distância do tijolo, $\mathrm{m} \mathrm{s}^{-1}$;

$\mathrm{V}_{2}$ - velocidade da onda ultra-sônica através da distância média do tijolo, $\mathrm{m} \mathrm{s}^{-1}$, e

$\mathrm{V}_{3}$ - velocidade da onda ultra-sônica através do comprimento do tijolo, $\mathrm{m} \mathrm{s}^{-1}$ (Figura 2).

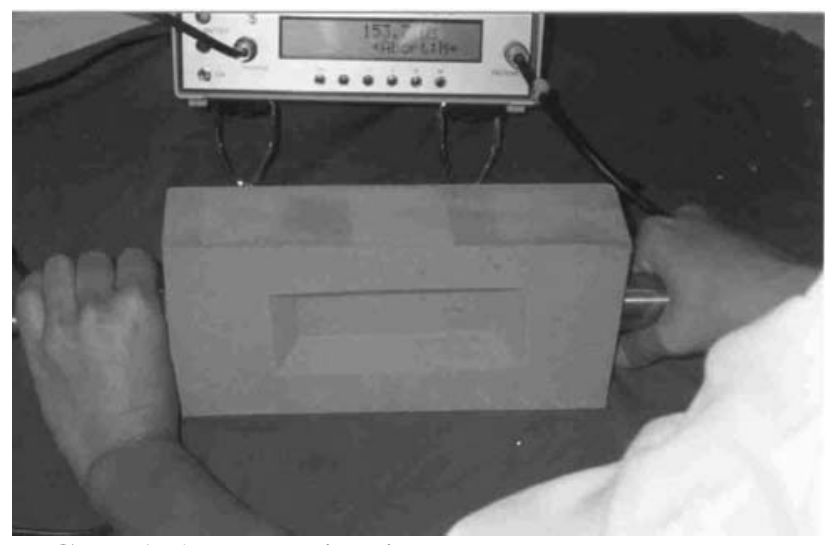

FIGURA 1. Teste do ultra-som.

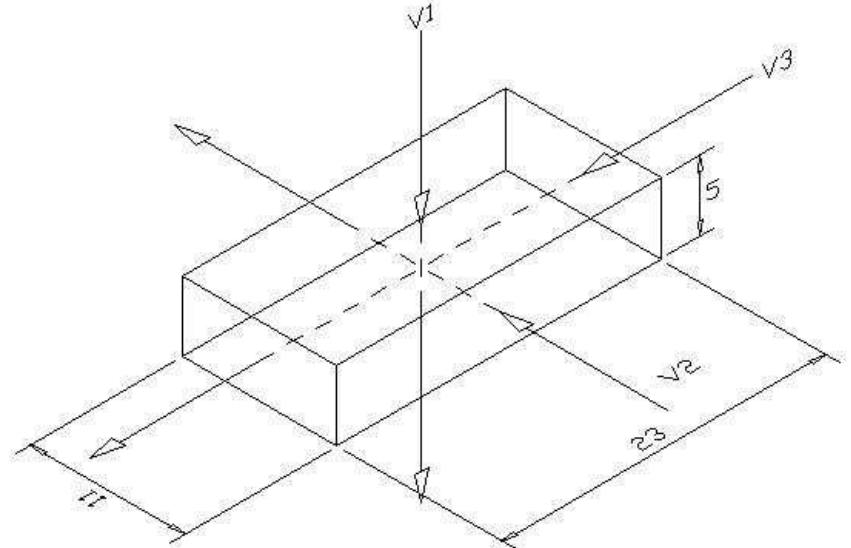

FIGURA 2. Direções de leitura do tempo de propagação da onda ultra-sônica. Dimensões em cm.

\section{Resistência anisotrópica}

Por meio da combinação dos resultados da resistência à compressão simples $\left(R_{C}\right)$ e da anisotropia total $(\Delta \mathrm{M})$, foi calculado o parâmetro quantitativo proposto por CULTRONE et al. (2001), denominado de resistência anisotrópica $\left(\mathrm{R}_{\mathrm{A}}\right.$, em $\mathrm{MPa}$ ) obtido mediante a eq.(5). Assim, maiores valores de $\mathrm{R}_{\mathrm{A}}$ indicam baixa anisotropia, geralmente associada à menor presença de espaços vazios $\mathrm{e}$ maior resistência mecânica.

$$
\mathrm{R}_{\mathrm{A}}=\frac{\mathrm{R}_{\mathrm{C}}}{\Delta \mathrm{M}}
$$

\section{Planejamento experimental}

$\mathrm{O}$ experimento foi conduzido em um delineamento inteiramento casualizado, em esquema fatorial $2 \times 9 \times 4$. Uma análise de variância de terceira ordem foi usada para estudar os efeitos das interações entre os fatores tipo de solo, adições químicas e idade de cura nos valores da variável resposta resistência anisotrópica. Para tal, foi empregado o pacote estatístico Statgraphics Plus 2.1®.

Todos os ensaios foram realizados em três repetições $(n=3)$. Embora $n=3$ seja estatisticamente aceito e a NBR 08492 recomende $n=10$ para os ensaios de compressão simples e absorção de água, os delineamentos experimentais em esquema fatorial são de difícil execução quando conduzidos em um número elevado de repetições. Portanto, devido às quantidades de tratamentos e idades de cura empregadas nesta pesquisa, optou-se por trabalhar com um número de repetições reduzido a fim de melhor garantir as mesmas condições de ensaio para todos os tratamentos. Ademais, trabalhos recentes relacionados à estabilização química de solos vêm adotando a prática de número de repetições reduzidas ( $n=3$ ou 4) sem prejuízos na explicação da variação dos efeitos proporcionados pelos tratamentos estudados (ALCÂNTARA et al., 1996; ROLIM \& FREIRE, 1998; ROLIM et al., 1999; VALENCIANO \& FREIRE, 2000; FERREIRA \& CAMARINI, 2001; FERREIRA \& FREIRE, 2003). 


\section{RESULTADOS E DISCUSSÃO}

Na Tabela 2, apresentam-se os valores de velocidade da onda ultra-sônica nas três direções dos tijolos destinados à ruptura por compressão simples. Houve aumento gradativo da velocidade em função do tempo devido ao processo de estabilização química dos tijolos, resultando em uma estrutura cristalina de melhor qualidade. De acordo com WALKER (1995) e WEBB (1992), uma estrutura cristalina de melhor qualidade se deve, principalmente, pelo preenchimento dos vazios pelos silicatos, aluminatos e carbonatos provenientes da hidratação do cimento e das reações de floculação e carbonatação entre a cal e a fração argila. $\mathrm{Na}$ Tabela 3, relacionam-se os valores de resistência à compressão simples, anisotropia total, resistência anisotrópica e capacidade de absorção de água obtidos dos ensaios destrutivos e não-destrutivos.

De maneira geral, os valores de resistência à compressão simples foram relativamente baixos e característicos de solos finos. Grandes quantidades de finos são responsáveis pelo enfraquecimento do efeito aglutinante entre a pasta de cimento e a fração arenosa do solo. Embora o cimento reaja até mesmo com solos finos e tende a estabilizá-los, a resistência da estrutura colóide/cimento é significativamente mais fraca que a estrutura granular/cimento. Da mesma forma, as condições de saturação em água durante o ensaio de compressão simples podem reduzir os valores da resistência. Segundo WALKER (1995), essa redução é atribuída ao desenvolvimento de pressões de água nos poros e à liquefação dos minerais de argila não estabilizados na matriz do tijolo.

Em termos de resistência à compressão, somente a adição de $10 \%$ de cimento para o solo arenoso (T5), aos 28 dias, e 10\% de cal para o solo siltoso (T16), aos 7 e 28 dias, atendeu às especificações da NBR 08491. Os elevados valores de resistência alcançados pelo solo siltoso natural (T10) se justificam pelo fato de os tijolos não terem sido saturados em água durante $4 \mathrm{~h}$, antes da ruptura, conforme recomendações da NBR 08492.

TABELA 2. Velocidade da onda ultra-sônica nas três direções dos tijolos.

\begin{tabular}{|c|c|c|c|c|c|c|c|c|c|c|c|c|}
\hline \multirow{3}{*}{ Trat. } & \multicolumn{12}{|c|}{ Velocidade $\left(\mathrm{m} \mathrm{s}^{-1}\right)$} \\
\hline & \multicolumn{4}{|c|}{$\mathrm{V}_{1}$} & \multicolumn{4}{|c|}{$\mathrm{V}_{2}$} & \multicolumn{4}{|c|}{$\mathrm{V}_{3}$} \\
\hline & 7 dias & 28 dias & 56 dias & 91 dias & 7 dias & 28 dias & 56 dias & 91 dias & 7 dias & 28 dias & 56 dias & 91 dias \\
\hline T1 & 874,17 & 991,82 & $1.017,43$ & 951,48 & 937,37 & $1.075,69$ & $1.119,83$ & $1.144,28$ & 922,55 & $1.080,55$ & $1.113,27$ & $.127,90$ \\
\hline $\mathrm{T} 2$ & $1.380,60$ & $1.418,16$ & $1.335,84$ & $1.234,83$ & $1.646,74$ & $1.696,70$ & $1.655,30$ & $1.575,24$ & $1.619,76$ & $1.651,93$ & $1.597,90$ & $1.530,04$ \\
\hline T3 & $1.698,86$ & $1.856,60$ & $1.848,25$ & $1.851,92$ & $1.979,61$ & $2.142,95$ & $2.171,29$ & $2.203,87$ & $1.950,29$ & $2.120,53$ & 0,89 & 33,64 \\
\hline T4 & 889,16 & $1.092,77$ & $1.103,00$ & 950,24 & $1.098,91$ & $1.407,14$ & $1.516,75$ & $1.240,62$ & $1.068,12$ & $1.318,17$ & 9,52 & 34,00 \\
\hline T5 & $1.710,73$ & $1.822,93$ & $1.375,92$ & $1.196,67$ & $2.001,36$ & $2.159,69$ & $1.877,54$ & $1.824,64$ & $2.039,01$ & $2.213,05$ & & 44,74 \\
\hline T6 & $1.009,09$ & $1.210,66$ & $1.265,94$ & $1.213,93$ & $1.191,93$ & $1.494,70$ & $1.589,34$ & $1.516,59$ & $1.170,97$ & $1.463,56$ & $1.498,36$ & \\
\hline $\mathrm{T} 7$ & 988,87 & $1.310,07$ & $1.396,74$ & $1.381,52$ & 7,71 & $1.559,30$ & $1.650,02$ & $1.664,33$ & $1.096,16$ & 5,50 & .79 & $1.593,27$ \\
\hline T8 & $1.069,86$ & 1.11 & 970,68 & & 1.2 & 9,33 & $1.188,95$ & & $1.254,98$ &, 48 & 24 & 1. \\
\hline T9 & 1.05 & 1.2 & 1.2 & & & 1.42 & 1.4 & & 1.19 & & & \\
\hline $\mathrm{T} 10$ & & 1.10 & 1.0 & & 2.1 & 2.8 & & & & & & \\
\hline T11 & 19 & $1.450,32$ & 1.24 & & 2.82 & 3.12 & $2.714,51$ & & 629,46 & 696,82 &, 54 & 90,6 \\
\hline T12 & $1.550,25$ & $1.518,29$ & $1.494,87$ & $1.485,18$ & $3.364,31$ & $3.350,26$ & $3.040,86$ & $3.193,63$ & 743,58 & 756,38 & 7,35 & 617,48 \\
\hline T13 & 857,79 & $1.071,17$ & $1.082,96$ & $1.046,86$ & $1.811,69$ & $2.145,25$ & $2.285,43$ & $2.349,16$ & 394,87 & 486,78 & 76,19 & 81,30 \\
\hline T14 & $1.103,82$ & $1.248,15$ & $1.333,45$ & $1.277,93$ & $2.579,04$ & $2.793,18$ & $2.987,32$ & $2.954,61$ & 559,54 & 600,53 & 2,87 & 21,77 \\
\hline T15 & $.425,22$ & $1.343,96$ & $1.065,39$ & $1.216,01$ & $3.028,19$ & $3.117,45$ & $2.698,08$ & $3.059,95$ & 671,24 & 674,28 & 3,13 & 638,6 \\
\hline T16 & 517,25 & $1.674,18$ & $1.501,52$ & $1.208,88$ & 3.21 & $3.819,44$ & $3.532,51$ & & 720,13 & 63,21 & 66 & 662,76 \\
\hline & & & & & & $2.529,39$ & $2.757,19$ & & & 44 & & \\
\hline T18 & $1.297,57$ & $1.319,25$ & $1.375,57$ & $1.267,19$ & $2.927,88$ & $2.965,86$ & $3.183,58$ & $3.427,02$ & 629,08 & 656,35 & 689,44 & 759,7 \\
\hline
\end{tabular}

Em termos de capacidade de absorção de água, todos os tratamentos aplicados aos tijolos arenosos atenderam às especificações da NBR 08491, que estabelece, como máximo, o valor médio de $20 \%$ e nenhum valor superior a $22 \%$ (Tabela 3). Entretanto, para o solo siltoso, somente a adição de $10 \%$ de cimento e silicato de sódio atendeu às especificações da NBR 08491. A principal explicação para esse comportamento é a alta porosidade dos tijolos siltosos, principalmente quando tratados com a 
cal, o que sugere outras estratégias de tratamento dos tijolos, como a impregnação e/ou adição de produtos hidrofugantes, como os estudados por REN \& KAGI (1995). De acordo com esses autores, a adição de soluções de silicato de sódio, silicone e siloxane reduziu consideravelmente a absorção de água de tijolos moldados a partir de solos finos.

Quanto à avaliação não-destrutiva, observa-se pelos valores da anisotropia total (Tabela 3) que os tijolos de solo arenoso foram menos anisotrópicos que os tijolos siltosos. Tais resultados podem ser explicados pela maior presença de espaços vazios nos tijolos siltosos que interferiram na propagação da onda ultra-sônica, originando diferenças significativas entre os valores de velocidade da onda ultrasônica nas três direções dos tijolos. Contudo, apesar da menor anisotropia total dos tijolos arenosos, os menores valores de resistência à compressão simples obtidos pelos mesmos acarretaram decréscimo nos valores da resistência anisotrópica, provocando, conseqüentemente, diminuição na qualidade dos tijolos.

TABELA 3. Valores de resistência à compressão simples $\left(R_{C}\right)$, anisotropia total $(\Delta M)$, resistência anisotrópica $\left(\mathrm{R}_{\mathrm{A}}\right)$ e absorção de água $(\mathrm{Ab})$ dos tijolos.

\begin{tabular}{ccccccccccccccc}
\hline \multirow{2}{*}{ Trat. } & \multicolumn{4}{c}{$\mathrm{R}_{\mathrm{C}}(\mathrm{MPa})$} & \multicolumn{4}{c}{$\Delta \mathrm{M}(\%)$} & \multicolumn{4}{c}{$\mathrm{R}_{\mathrm{A}}(\mathrm{MPa} / \%)^{*}$} & \multicolumn{3}{c}{$\mathrm{Ab}(\%)$} \\
\cline { 2 - 15 } & 7 dias & 28 dias & 56 dias & 91 dias & 7 dias & 28 dias & 56 dias & 91 dias & 7 dias & 28 dias & 56 dias & 91 dias & 7 dias \\
\hline T1 & 0,95 & 0,66 & 0,64 & 0,61 & 0,60 & 0,79 & 0,89 & 1,63 & 0,16 & 0,09 & 0,07 & 0,04 & - \\
T2 & 0,77 & 0,83 & 0,72 & 0,68 & 1,55 & 1,53 & 1,79 & 2,05 & 0,05 & 0,06 & 0,04 & 0,03 & 11,86 \\
T3 & 1,39 & 1,84 & 1,84 & 1,40 & 1,35 & 1,29 & 1,47 & 1,48 & 0,10 & 0,14 & 0,13 & 0,10 & 11,53 \\
T4 & 0,42 & 1,02 & 1,22 & 1,02 & 1,79 & 1,99 & 2,36 & 2,16 & 0,02 & 0,05 & 0,05 & 0,05 & 14,43 \\
T5 & 1,84 & 2,03 & 1,90 & 1,07 & 1,53 & 1,66 & 2,76 & 3,24 & 0,12 & 0,12 & 0,07 & 0,03 & 13,84 \\
T6 & 0,54 & 0,74 & 0,79 & 0,67 & 1,46 & 1,82 & 1,80 & 1,85 & 0,04 & 0,04 & 0,04 & 0,04 & 13,79 \\
T7 & 0,45 & 0,76 & 0,92 & 0,70 & 1,18 & 1,48 & 1,46 & 1,52 & 0,04 & 0,05 & 0,06 & 0,05 & 13,40 \\
T8 & 0,64 & 0,57 & 0,36 & 0,30 & 1,43 & 1,82 & 1,78 & 2,04 & 0,05 & 0,03 & 0,02 & 0,02 & 15,43 \\
T9 & 0,66 & 0,53 & 0,83 & 0,76 & 1,18 & 1,40 & 1,49 & 1,93 & 0,06 & 0,04 & 0,06 & 0,04 & 16,06 \\
T10 & 0,77 & 1,89 & 2,53 & 2,41 & 4,12 & 3,66 & 3,54 & 3,86 & 0,02 & 0,05 & 0,07 & 0,06 & - \\
T11 & 0,50 & 0,39 & 0,37 & 0,45 & 2,04 & 2,40 & 2,44 & 3,10 & 0,03 & 0,02 & 0,02 & 0,02 & 26,09 \\
T12 & 1,01 & 0,67 & 0,60 & 0,82 & 2,45 & 2,60 & 1,97 & 2,20 & 0,04 & 0,03 & 0,04 & 0,04 & 25,21 \\
T13 & 0,35 & 0,33 & 0,49 & 0,51 & 2,22 & 1,86 & 2,15 & 2,60 & 0,02 & 0,02 & 0,02 & 0,02 & 23,72 \\
T14 & 0,46 & 0,76 & 0,81 & 0,89 & 2,97 & 2,64 & 2,61 & 2,85 & 0,02 & 0,03 & 0,03 & 0,03 & 19,97 \\
T15 & 1,52 & 1,17 & 0,60 & 0,69 & 2,29 & 2,91 & 3,51 & 3,43 & 0,07 & 0,04 & 0,02 & 0,02 & 26,01 \\
T16 & 2,00 & 2,14 & 1,08 & 1,08 & 2,29 & 2,84 & 3,03 & 3,61 & 0,09 & 0,08 & 0,04 & 0,03 & 27,09 \\
T17 & 0,84 & 0,67 & 0,82 & 0,30 & 2,85 & 2,71 & 3,55 & 3,89 & 0,03 & 0,03 & 0,02 & 0,01 & 25,70 \\
T18 & 1,07 & 1,39 & 1,18 & 0,76 & 2,70 & 2,71 & 2,90 & 3,95 & 0,04 & 0,05 & 0,04 & 0,02 & 23,50 \\
\hline
\end{tabular}

*Dados usados na análise de variância.

A análise de variância revelou que todos os fatores, bem como suas interações, foram significativos na influência dos valores da resistência anisotrópica $\left(\mathrm{R}_{\mathrm{A}}\right)$ (Tabela 4). A comparação das médias dos valores de $\mathrm{R}_{\mathrm{A}}$ sob a influência dos fatores tipo de solo, adição química e tempo de cura evidenciou diferenças significativas, a 5\% de probabilidade (Tabela 5).

Em relação ao tipo de solo, o siltoso foi superior ao arenoso em termos de resistência anisotrópica. Quanto às adições químicas, as que proporcionaram a maior e a menor qualidade foram as de $10 \%$ de cimento e $6 \%$ de cal associada ao silicato de sódio, respectivamente. Os valores da resistência anisotrópica tenderam a diminuir com o aumento da idade. Tal efeito leva a crer que o processo de cura favoreceu o aumento da anisotropia total decorrente do incremento das diferenças entre os valores de velocidades da onda ultra-sônica nas três direções dos tijolos (Tabela 5).

Portanto, o uso da resistência anisotrópica como parâmetro para avaliar a qualidade dos tijolos baseou-se na relação entre o estresse físico produzido no tijolo por ocasião do ensaio de compressão simples e um parâmetro matemático (sua anisotropia estrutural). Assim, em consonância com 
CULTRONE et al. (2001), quanto maior o valor da resistência anisotrópica, melhor foi a qualidade do material, ou seja, a qualidade dos tijolos ficou condicionada a sua estrutura anisotrópica para um estresse mecânico constante.

TABELA 4. Análise de variância para a variável-resposta resistência anisotrópica (em MPa/\%).

\begin{tabular}{lcccc}
\hline Fontes de Variação & GL & SQ & QM & F \\
\hline Principais efeitos & & & & \\
A: solo & 1 & 0,0387 & 0,0387 & $360,00^{*}$ \\
B: adições químicas & 8 & 0,0635 & 0,0079 & $73,87^{*}$ \\
C: idade & 3 & 0,0132 & 0,0044 & $41,12^{*}$ \\
\hline Interações & & & & \\
A X B & 8 & 0,0404 & 0,0050 & $46,98^{*}$ \\
A X C & 3 & 0,0026 & 0,0009 & $8,19^{*}$ \\
B X C & 24 & 0,0107 & 0,0004 & $4,14^{*}$ \\
A X B X C & 24 & 0,0433 & 0,0018 & $16,79^{*}$ \\
\hline Resíduo & 144 & 0,0155 & 0,0001 & \\
\hline Total & 215 & 0,2278 & & \\
\hline
\end{tabular}

*Significativo a 5\% de probabilidade.

TABELA 5. Análise do efeito da interação entre os fatores tipo de solo, adição química e idade nos valores médios da resistência anisotrópica (em MPa/\%).

\begin{tabular}{|c|c|c|c|}
\hline Fatores & Níveis & Observações* & Médias \\
\hline \multirow{2}{*}{ Solos } & Siltoso & 108 & $0,061 \mathrm{a}$ \\
\hline & Arenoso & 108 & $0,034 \mathrm{~b}$ \\
\hline \multirow{9}{*}{ Adição química } & $10 \%$ de cimento & 24 & $0,079 \mathrm{a}$ \\
\hline & Solo natural & 24 & $0,072 \mathrm{a}$ \\
\hline & $10 \%$ de cimento $+4 \%$ de silicato de sódio & 24 & $0,056 \mathrm{~b}$ \\
\hline & $10 \%$ de cal & 24 & $0,054 \mathrm{~b}$ \\
\hline & $10 \%$ de cal $+4 \%$ de silicato de sódio & 24 & $0,043 \mathrm{c}$ \\
\hline & $6 \%$ de cal & 24 & $0,038 \mathrm{~cd}$ \\
\hline & $6 \%$ de cimento $+4 \%$ de silicato de sódio & 24 & $0,031 \mathrm{de}$ \\
\hline & $6 \%$ de cimento & 24 & $0,031 \mathrm{de}$ \\
\hline & $6 \%$ de cal $+4 \%$ de silicato de sódio & 24 & $0,025 \mathrm{e}$ \\
\hline \multirow{4}{*}{ Idade } & 7 dias & 54 & $0,055 \mathrm{a}$ \\
\hline & 28 dias & 54 & $0,053 \mathrm{a}$ \\
\hline & 56 dias & 54 & $0,046 \mathrm{~b}$ \\
\hline & 90 dias & 54 & $0,035 \mathrm{c}$ \\
\hline
\end{tabular}

* Número de observações correspondentes a três repetições para cada nível dentro de cada fator (solo, adição química e idade); ** Médias seguidas de mesma letra não diferem entre si, pelo teste de Tukey, a $5 \%$ de probabilidade.

\section{CONCLUSÕES}

Os efeitos dos fatores tipo de solo, adições químicas e período de cura, bem como suas interações, proporcionaram diferenças significativas nos valores da resistência anisotrópica dos tijolos.

No geral, os tijolos arenosos foram menos anisotrópicos que os siltosos, efeito devido principalmente à maior porosidade dos tijolos siltosos. Todavia, a baixa anisotropia dos tijolos arenosos não confirmou sua melhor qualidade técnica, visto que seus valores de resistência mecânica foram relativamente baixos. 
Os tijolos que obtiveram a pior e a melhor qualidade técnica, avaliada pela resistência anisotrópica, foram aqueles adicionados de $6 \%$ de cal associada ao silicato de sódio e $10 \%$ de cimento, respectivamente.

Os resultados obtidos permitem afirmar que a qualidade dos tijolos pode ser avaliada mediante a combinação dos efeitos do estresse mecânico e da estrutura anisotrópica dos mesmos, e confirma a "resistência anisotrópica" como um parâmetro quantitativo viável para o uso no controle e na avaliação da qualidade de tijolos de terra crua estabilizados quimicamente.

\section{REFERÊNCIAS}

ALCÂNTARA, M.A.M.; SILVA, S.A.M.; AGUILLAR FILHO, D.; SEGANTINI, A.A.S. Estabilização de solos com cal em construções rurais. In: CONGRESSO BRASILEIRO DE ENGENHARIA AGRÍCOLA, 25., 1996, Bauru. Anais... Bauru: Sociedade Brasileira de Engenharia Agrícola, 1996. 1 CD ROM.

ASSOCIAÇÃO BRASILEIRA DE NORMAS TÉCNICAS. NBR 06459: Solo: Determinação do limite de liquidez. Rio de Janeiro, 1984a. 6p. NBR 07175: Cal hidratada para argamassas. Rio de Janeiro, 1992a. 3p. NBR 07180: Solo: Determinação do limite de plasticidade. Rio de Janeiro, 1984b. 3p. . NBR 07181: Solo: Análise granulométrica. Rio de Janeiro, 1984c. 13p. NBR 07182: Solo: Ensaio de compactação. Rio de Janeiro, 1986. 10p. NBR 07251: Agregado em estado solto: Determinação da massa específica unitária.. Rio de Janeiro, 1982. 3p. NBR 08491: Tijolo maciço de solo-cimento. Rio de Janeiro, 1992b. 8p. NBR 08492: Tijolo maciço de solo-cimento: Determinação da resistência à compressão. Rio de Janeiro, 1992c. 8p.

NBR 10832: Fabricação de tijolo maciço de solo-cimento com a utilização de prensa manual. Rio de Janeiro, 1992d. 8p. . NBR 11578: Cimento Portland CPII-E-32. Rio de Janeiro, 1991. 5p.

BARBOSA, N.P.; MATTONE, R. Estudos sobre tijolos de terra crua desenvolvidos na Universidade Federal da Paraíba e no Politécnico de Torino. In: CONGRESSO DE ENGENHARIA CIVIL, 2., 1996, Juiz de Fora. Anais... Juiz de Fora: UFJF, 1996. v.1, p.21-30.

BARONIO, G; BINDA, L. Study of the pozzolanicity of some bricks and clays. Constructions and Building Materials, Guildford, v.11, n.1, p.41-6. 1997.

BERALDO, A.L. Ensaios de caracterização mecânica de materiais de construção. Disponível em: <ftp://ftp.agr.unicamp.br/pub/disciplinas/ap152/> Acesso em: 20 jun. 2002.

CULTRONE, G.; SEBASTIÁN, E; CAZALlA O.; NECHAR, M.; ROMERO, R.; BAGUR, M.R. Ultrasound and mechanical tests combined with ANOVA to evaluate brick quality. Ceramics International, Faenza, v.27, p.401-6, 2001.

CAZALlA, O; SEBASTIÁN, E.; CULTRONE, G.; NECHAR, M.; BAGUR, M.G. Three way ANOVA interaction analysis and ultrasonic testing to evaluate air lime mortars used in cultural heritage conservation projects. Cement and Concrete Research, Elmsford, v.29, p.1749-52, 1999. FERREIRA, R.C.; CAMARINI, G. Compressive strength of soil stabilized with lime through ultrasonic method. In: INTERNATIONAL SYMPOSIUM ON AGRICULTURAL BUILDING AGRIBUILDING, 3., 2001, Campinas. Proceedings... Campinas: CIGR, 2001. 1 CD ROM. 
FERREIRA, R.C.; FREIRE, W.J. Propriedades físico-mecânicas de solos estabilizados com cimento e silicato de sódio avaliadas por meio de testes destrutivos e não-destrutivos. Engenharia Agrícola, Jaboticabal, v.23, n.2, p.221-32, 2003.

FREIRE, W.J. Aditivos químicos e seus efeitos sobre a estabilidade de taludes e qualidade do material formado. 1981. 113 f. Tese (Livre-Docência) - Faculdade de Ciências Agronômicas, Universidade Estadual Paulista Júlio de Mesquita Filho, Botucatu, 1981.

GEYER, R.T.; MASUERO, A.B.; VILELA, A.C.F.; DAL MOLIN, D.C.C. Aproveitamento de escórias siderúrgicas na construção civil. In: JORNADAS SUDAMERICANAS DE INGENIERIA ESTRUCTURAL, 27., 1995, Tucumán, Argentina. Anais ... p.423-34.

GORDON, J.N.; PINNOCK, W.R.; MOORE, M.M. A preliminary investigation of strentth development in Jamaican red mud composites. Cement and Concrete Composites, Barking, v.18, p.371-9, 1996.

KANIRAJ, S.R.; HAVANAGI, V.G. Compressive strength of cement stabilized fly-ash-soil mixtures. Cement and Concrete Research, Elmsford, v.29, n.5, p.673-7, 1999.

NGOWI, A.B. Improving the traditional earth construction: a case study of Botswana. Construction and Building Materials, Guildford, v.11, n.1, p.1-7, 1997.

QASRAWI, H.Y. Concrete strength by combined nondestructive methods simply e reliably predicted. Cement and Concrete Research, Elmsford, v.30, p.739-46, 2000.

REN, K.B.; KAGI, D.A. Upgrading the durability of mud bricks by impregnation. Building and Environment, Oxford, v.30, n.3, p.433-40, 1995.

ROLIM, M.M.; FREIRE, W.J. Resistência à compressão de tijolos fabricados com solo-vinhaça concentrada. Engenharia Agrícola, Jaboticabal, v.17, n.3, p.1-8, 1998.

ROLIM, M.M.; FREIRE, W.J.; BERALDO, A.L. Análise comparativa da resistência à compressão simples de corpos de prova, tijolos e painéis de solo-cimento. Revista Brasileira de Engenharia Agrícola e Ambiental, Campina Grande, v.3, n.1, p.93-8, 1999.

RUFF,C.G.; DAVIDSON, D.T. Lime and sodium silicate stabilization of montmorillonite clay soil. Highway Research Board Bulletin, Washington, v.304, n.1, p.76-92, 1961.

SHERWOOD, P.T. Soil stabilization with cement and lime: state of the art review. London: HMSO, 1993. 153p.

VALENCIANO, M.C.M.; FREIRE, W.J. Características físico-mecânicas de tijolos de solo melhorado com cimento e fibras de bagaço de cana-de-açúcar. In: CONFERENCIA CIENTIFICO-TECNICA DE LA CONSTRUCCIÓN, 4., 2000, La Habana - Cuba. Editorial de la Construcción, 2000. p.179-83.

WALKER, P.J. Strength, durability and shrinkage characteristics of cement stabilized soil blocks. Cement and Concrete Composites, Barking, v.17, n.4, p.301-10, 1995.

WEBB, D.J.T. Lime stabilized soil blocks for third world housing. In: HILL, N.; HOLMES, S.; MATHER, D. Lime and other alternative cements. London: Intermediate Technology Publications, 1992. p.246-57. 